\title{
Weight development from age 13 to 30 years and adolescent socioeconomic status: The Norwegian Longitudinal Health Behaviour study
}

\author{
Ingunn Holden Bergh • Øivind Skare • Annalena Aase • \\ Knut-Inge Klepp $\cdot$ Nanna Lien
}

Received: 30 January 2015/Revised: 15 September 2015/ Accepted: 24 September 2015/Published online: 7 October 2015

(C) The Author(s) 2015. This article is published with open access at Springerlink.com

\begin{abstract}
Objectives To describe the weight development and model change in body mass index (BMI), and to examine the association of adolescent socioeconomic status (SES) with change in BMI distribution in a cohort followed from adolescence through adulthood.

Methods Participants $(n=924)$ from western Norway were surveyed seven times from age 13 to 30 (1990-2007). BMI was based on self-reported height and weight. Quantile regression analyses were used to model change in weight development and to investigate associations between SES (measured by parental education) and change in BMI distribution. The analyses were adjusted for curvilinearity in BMI development, gender and relevant health behaviours.

Results Body mass index increased over time with the greatest increase in the 90th percentile. No significant associations between change in BMI and SES were observed at any of the percentiles (10th, 25th, 50th, 75th or 90th).

Conclusions Those in the upper BMI percentile gained more weight than those in the lower percentiles indicating
\end{abstract}

\footnotetext{
I. H. Bergh · Ø. Skare · A. Aase · K.-I. Klepp · N. Lien $(\bowtie)$ Department of Nutrition, Faculty of Medicine, University of Oslo, P.O. Box 1046, Blindern, 0316 Oslo, Norway

e-mail: nanna.lien@medisin.uio.no

I. H. Bergh

e-mail: i.h.bergh@medisin.uio.no

$\emptyset$. Skare

e-mail: oivind.skare@medisin.uio.no

A. Aase

e-mail: annalenaase@ hotmail.com

K.-I. Klepp

e-mail: k.i.klepp@medisin.uio.no
}

that these might need targeted interventions. Further investigation of the association of change in BMI and SES with better quality data might be warranted.

Keywords Body mass index · Adolescents - Adults · Weight gain $\cdot$ Socioeconomic status

\section{Introduction}

The prevalence of overweight and obesity in children and adults in Europe is a public health concern (Berghofer et al. 2008; Brug et al. 2012). Overweight children do often become overweight or obese adults, but many develop their overweight/obesity after childhood (Power et al. 1997). In addition to gestation and early infancy and the period of adiposity rebound, adolescence is a critical periods for obesity development (Dietz 1994). While an increase in adiposity in early life may be associated with normal growth, an increase in adiposity after growth has stabilized (18-20 years) is more likely to be a threat to health (Dietz 1994; Power et al. 1997).

Norwegian studies show consistently higher levels of overweight and obesity among adults than in children and adolescents (Bjelland et al. 2010; Cuypers et al. 2012; Juliusson et al. 2010; Meyer and Tverdal 2005). The increase in overweight and obesity in recent decades seems to be a consequence of a larger weight gain over time among those with a higher BMI to begin with (Ekblom et al. 2004; Kautiainen et al. 2002). This indicates that those in the upper part of the body mass index (BMI) distribution may be more vulnerable to influences on weight gain.

An inverse relationship between socioeconomic status (SES) and body mass index (BMI) has long been 
recognized as a public health challenge (Ball and Crawford 2005). Among adults low SES is associated with greater weight gain than higher SES, and SES differences in weight development may differ by gender (Ball and Crawford 2005; Giskes et al. 2008). However, a differential rate of weight gain influenced by SES possibly begins early in life (Brisbois et al. 2012), and differences in adiposity and prevalence of obesity in adulthood seem to stem, at least partly, from the socioeconomic circumstances of the family (Ball and Crawford 2005; Giskes et al. 2008; Hardy et al. 2000).

Still, knowledge about the influence of adolescent SES on weight development is limited. Longitudinal studies with cohorts encompassing childhood/adolescence through adulthood with long follow-up periods have also been called for (Giskes et al. 2008; Pate et al. 2013; Tamayo et al. 2010). Furthermore, no study has investigated whether those in the upper part of the BMI distribution are more vulnerable to a possible influence of SES on weight change over time than those in the lower part of the distribution. A possible accelerated weight gain among low SES individuals in the upper part of the BMI distribution may compound existing health inequalities.

Some of the most recent longitudinal studies examining influences on weight development have provided evidence of the utility of quantile regression (Bottai et al. 2014; Mitchell et al. 2013a, b, c). In contrast to modelling the mean change, this analytic approach allows for a specific investigation of the tails of the BMI which is of particular interest in the realm of public health (Hao and Naiman 2007). Hence, the aim of this study was twofold: to describe the weight development and to model change in the BMI distribution in a cohort with young Norwegian adolescents followed into adulthood, and to examine whether adolescent SES was associated with change in the BMI distribution over time.

\section{Methods}

Sample

The data stem from the 17-year Norwegian Longitudinal Health Behaviour (NLHB) study among adolescents and their parents/guardians. Participants were recruited from 22 randomly selected schools in the county of Hordaland, Norway. At baseline in 1990, the sample included a representative sample of 924 students from 7 th grade (78\% response rate). Questionnaires were administered in October through school at age 13-15 (1990-1992), and thereafter by mail to the participants' home address at age 16 (1993), 18 (1995), 19 (1996), 21 (1998), 23 (2000) and age 30 (2007). A parental/guardian survey was administered in 1996. Written consent from parents/guardians and the adolescents was given prior to the study.

A detailed description of the sampling procedures and data collection in the NLHB study is presented previously (Lien et al. 2001b). The study was approved by the Norwegian Data Inspectorate. It has been conducted in accordance with ethical principles, including the provisions of the World Medical Associations Declaration of Helsinki.

\section{Measures}

Body mass index $\left(\mathrm{kg} / \mathrm{m}^{2}\right)$ was calculated from the participants' self-reported height and weight at all ages (except at age 16 when participants were not asked about this). Overweight and obesity prevalence, presented for descriptive purposes, were calculated based on International Obesity Task Force's cut-points for the participants up until age 18 (Cole and Lobstein 2012) and by the adult World Health Organization cutoffs from age 18 (WHO 2000). The BMI data for pregnant women at age 23 $(n=7)$ and $30(n=14)$ (only asked for at these ages) were excluded.

The participants' parents reported their highest level of education in 1996 (adolescents age 19). The adolescents were asked about the highest level of education for each of their parents in 1992 (adolescents age 15). The pre-coded answers were collapsed into the following categories: elementary school (no education beyond 9 years of mandatory school), upper secondary school (1-3 years of upper secondary school) and college/university (1 year or more of college/university), further labelled low, medium and high SES groups, respectively. When parental reported data for years of education were missing (42.2\%), the educational variable was supplemented with the adolescents' response (30\%), as used previously (Lien et al. 2001a). The data from the parent with the highest reported education level or the one available were used.

Gender, soda, chocolate/sweet and breakfast consumption, physical activity and smoking habits were included as covariates. Frequency of consuming (1) sugar containing soda, (2) chocolate/sweets, and (3) breakfast was assessed by frequency questions "How often do you drink eat/drink....?" The response categories with the recoding to times per week in parentheses were for the (1) soda item: not every week (0.5); 1-2 times per week (1.5); 3-6 times per week (4.5); 1 time per day (7); greater than 1 time per day (10); for the (2) chocolate/sweets item which were assumed to be eaten more rarely: never (0); and seldom (1); 1-2 times per week (1.5); 3-6 times per week (4.5); every day (7), for the (3) breakfast item: not that often (0.5); 1-3 times per week (1.5); 4-6 times per week (5); every day (7) (Lien et al. 2001b). 
Physical activity was assessed using the question: "Outside school hours (or outside work hours), how many hours per week do you do sport or exercise until you are out of breath or sweat"? The response categories with the recoding to $\mathrm{h} /$ week in parentheses were: none $(0)$; about $1 / 2$ h/week (0.5); about 1 h/week (1); about 2-3 h/week (2.5); about 4-6 h/week (5); $7 \mathrm{~h}$ or more (7) (Anderssen et al. 1995).

Smoking was assessed by the question: "How often do you smoke?" with the following response categories: every day, every week, less than once a week, and collapsed into the following ordinal levels: not smoking (1); occasional smoking (2); regular smoking (3) (Friestad and Klepp 1997).

\section{Statistical analyses}

Descriptive statistics are presented with means and SDs for continuous variables while frequencies and percentages are used for categorical variables. Quantile regression was used for the longitudinal analyses. This approach is an extension of ordinary least square regression and models the effect of predictors across the distribution of a continuous dependent variable (Hao and Naiman 2007; Wei et al. 2006). The coefficients from the quantile regression are interpreted in the same manner as in ordinary least square regression (i.e., change in the outcome variable for each one-unit change in the predictor) (Hao and Naiman 2007). All participants having at least one BMI observation were included in the quantile regression analyses. Observations with non-complete covariate information were, however, excluded prior to analysis. In model 0 , BMI was entered as the dependent variable, with study age and gender included as covariates, to describe changes in the BMI distribution over time, specified to the 10th, 25th, 50th, 75th and 90th BMI percentiles. The age variable was centred at age 13 to facilitate the interpretation of model coefficients. An interaction term between age and gender (age $\times$ gender) allowed for different linear age trends for male and females. In model 1 , an age ${ }^{2}$ term was included to investigate if changes in BMI were linear or curvilinear, and an age $^{2} \times$ gender term was included to examine whether any curvilinearity varied by gender over time. In model 2 , parental education level was added as the predictor of interest, interacting with age (age $\times$ SES), to examine if adolescent SES was associated with changes in the BMI distribution over time, keeping the covariates from model 1. Next, in model 3a, the dietary behaviours were added. In model $3 \mathrm{~b}$, physical activity was added. Finally, in model $3 \mathrm{c}$, smoking level was added. In these three models, all added covariates were interacting with age such that the effect of each covariate could vary with age. The behavioural variables were entered step-wise to investigate whether any association between adolescent SES and changes in BMI remained when adjusting for these groups of covariates. As a last step, the moderating effect of gender in the association between SES and change in BMI (age $\times$ gender $\times$ SES) was investigated. Quantile regression assumes independent observations. As we have dependency in the date due to repeated measurements, standard deviations, and therefore also $p$ values, reported from this analysis will generally be biased. Robust standard deviations were then estimated by bootstrapping using a case resampling scheme with 1000 replications (Wei et al. 2006). To retain the dependency structure in the bootstrap samples, we sampled subjects, not individual observations. This means that all observations of the sampled subjects were included in the bootstrap data.

The quantile regression analyses were conducted in Stata (version 13, College Station, StataCorp LP, Texas, USA). All other analyses were done in IBM SPSS (version 19, IBM Corp., Somers, New York, USA).

\section{Results}

At baseline (age 13), there were slightly more boys than girls (55 vs $45 \%)$. The proportion with low, medium and high SES was 14, 44 and $42 \%$, respectively. Participation rate at age 14,15,18,19, 21, 23 and 30 was $96.3 \%$ (890), $94.0 \%$ (869), $71.0 \%$ (656), $61.6 \%$ (569), $60.2 \%$ (556), $58.2 \%$ (538), $50.3 \%$ (465), respectively. Height and weight were reported from $80.7 \% 82.8 \%, 92.5,83.2$, $96.0,91.9,95.0$, and $93.6 \%$ at age $13,14,15,18,19,21$, 23 and 30, respectively. There were no differences between those without and with BMI at baseline and follow-up at age 14 and 15 in the gender and SES distribution, but those who were missing BMI due to either missing weight and/or height data were more likely to be boys and to belong to the low SES group at age 18, 21, 23 and 30.

Table 1 illustrates that BMI was significantly lower among females than males from age 15 and upwards. The descriptive data points to a steady increase in BMI from age 13-30 for both genders. At the last follow-up half of the men were either overweight or obese compared to about one-third of the women. Both among women and men, the descriptive data shows that less than $10 \%$ of the sample were obese at age 30 , but within the overweight/ obese groups the relative proportion with obesity were higher among women $(27 \%)$ than men $(16 \%)$.

Table 2 shows a trend for average BMI being higher among those with low vs high SES at each age with the difference being significant at age 21 and 30 . At age 14 and 18 , there was a significant difference between those with medium vs high SES. There were also differences between the SES groups for the proportions of overweight/obese, 
Table 1 Body mass index (BMI), proportion overweight/obese and obese at baseline and follow-ups in the Norwegian Longitudinal Health Behaviour study (1990-2007), all and by gender

\begin{tabular}{|c|c|c|c|c|c|c|c|}
\hline & $n$ & All & $n$ & Females & $n$ & Males & $p^{\mathrm{a}, \mathrm{b}}$ \\
\hline \multicolumn{8}{|c|}{ BMI, mean (SD) } \\
\hline Age 13 & 746 & $18.2(2.1)$ & 335 & $18.3(2.2)$ & 411 & $18.2(2.1)$ & 0.487 \\
\hline Age 14 & 737 & $19.2(2.2)$ & 319 & $19.2(2.2)$ & 418 & $19.2(2.2)$ & 0.601 \\
\hline Age 15 & 804 & $20.1(2.3)$ & 359 & $19.9(2.1)$ & 445 & $20.3(2.5)$ & 0.021 \\
\hline Age 18 & 613 & $21.7(2.6)$ & 309 & $21.3(2.6)$ & 304 & $22.1(2.5)$ & $<0.001$ \\
\hline Age 19 & 546 & $22.1(2.6)$ & 283 & $21.7(2.8)$ & 263 & $22.5(2.4)$ & $<0.001$ \\
\hline Age 21 & 501 & $22.6(2.8)$ & 269 & $22.1(2.9)$ & 232 & $23.2(2.6)$ & $<0.001$ \\
\hline Age 23 & 511 & $23.1(3.3)$ & 255 & $22.5(3.5)$ & 256 & $23.7(2.9)$ & $<0.001$ \\
\hline Age 30 & 435 & $24.7(3.6)$ & 220 & 23.9 (3.9) & 215 & $25.5(3.1)$ & $<0.001$ \\
\hline \multicolumn{8}{|c|}{$\begin{array}{l}\text { Overweight/ } \\
\text { obese, \% (n) }\end{array}$} \\
\hline Age 13 & 746 & $4.6(34)$ & 335 & $4.2(14)$ & 411 & $4.9(20)$ & 0.786 \\
\hline Age 14 & 737 & $5.2(38)$ & 319 & $4.1(13)$ & 418 & $6.0(25)$ & 0.322 \\
\hline Age 15 & 804 & $7.6(61)$ & 359 & $4.2(15)$ & 445 & $10.3(46)$ & 0.002 \\
\hline Age 18 & 613 & $8.5(52)$ & 309 & $6.8(21)$ & 304 & $10.2(31)$ & 0.172 \\
\hline Age 19 & 546 & $11.4(62)$ & 283 & $11.0(31)$ & 263 & $11.8(31)$ & 0.864 \\
\hline Age 21 & 501 & $16.4(82)$ & 269 & $15.2(41)$ & 232 & $17.7(41)$ & 0.540 \\
\hline Age 23 & 511 & $24.3(124)$ & 255 & $18.4(47)$ & 256 & 30.1 (77) & 0.003 \\
\hline Age 30 & 435 & $41.6(181)$ & 220 & $32.7(72)$ & 215 & 50.7 (109) & $<0.001$ \\
\hline \multicolumn{8}{|c|}{ Obese, \% (n) } \\
\hline Age 13 & 746 & $0.3(2)$ & 335 & NA & 411 & $0.5(2)$ & NA \\
\hline Age 14 & 737 & $0.4(3)$ & 319 & $0.3(1)$ & 418 & $0.5(2)$ & NA \\
\hline Age 15 & 804 & $0.6(5)$ & 359 & NA & 445 & $1.1(5)$ & NA \\
\hline Age 18 & 613 & $1.0(6)$ & 309 & $0.6(2)$ & 304 & $1.3(4)$ & NA \\
\hline Age 19 & 546 & $1.5(8)$ & 283 & $1.8(5)$ & 263 & $1.1(3)$ & NA \\
\hline Age 21 & 501 & $2.0(10)$ & 269 & $1.5(4)$ & 232 & $2.6(6)$ & NA \\
\hline Age 23 & 511 & 3.7 (19) & 255 & $4.3(11)$ & 256 & $3.1(8)$ & NA \\
\hline Age 30 & 435 & $8.3(36)$ & 220 & $8.6(19)$ & 215 & 7.9 (17) & NA \\
\hline
\end{tabular}

NA not applicable

a Difference between genders tested with independent $t$ and Chi-square test for continuous and categorical variables, respectively

${ }^{\mathrm{b}}$ Due to low numbers of obese cases, differences between gender are not statistically analysed

with a trend for a higher prevalence among those with low vs high SES at each age (non-significant at age 19 and 23).

Table 3 shows the results from the quantile regression analyses. In model 0 , the coefficient for the age term gives the average yearly increase in BMI for males, while the coefficient for the age $\times$ gender interaction term gives the difference in yearly increase between females and males. The average increase per year in BMI over the 17-year time period in the 90th percentile was 0.54 vs 0.38 in the 10th percentiles for males, and $0.50 \mathrm{BMI}$ units vs 0.23 for females. This corresponds to a total increase in BMI over the 17-year period of 9.17 in the 90th percentile vs 6.47 in the 10th percentile for males and of 8.47 vs 3.90 for females.

Model 1 shows that the change in BMI was curvilinear for males $\left(\mathrm{age}^{2}\right)$ in all the BMI percentiles and the rate of change decelerated with increasing age. The deceleration in the rate of change was smaller among females $\left(\right.$ age $^{2} \times$ gender) in the 25th, 50th and 90th percentile (borderline significant). The coefficient for the age term gives the initial increase at age 13 in BMI per year for males. In model 1, a higher rate of initial increase in BMI per year was observed in the upper vs lower tail of the distribution with increasingly larger coefficients (age) for each percentile. For example at the 90th BMI percentile the initial increase for males was $0.89(95 \%$ CI $0.75,1.03$ ) BMI-units per year (age) compared to 0.65 $(95 \%$ CI $0.57,07.4)$ at the 10 th percentile. The rate of initial increase in BMI per year was significantly lower in females (age $\times$ gender) than males in each percentile, except for the lowest and upper most percentile.

Model 2 shows that there were no significant association between SES and change in BMI. However, the association was strongest in the upper most percentile (90th); predicted 
Table 2 Body mass index (BMI) and proportion overweight/obese at baseline and follow-ups by socioeconomic status (SES) in the Norwegian Longitudinal Health Behaviour study (1990-2007)

\begin{tabular}{|c|c|c|c|c|c|c|c|}
\hline & $n^{\mathrm{a}}$ & Low SES & $n^{\mathrm{a}}$ & Medium SES & $n^{\mathrm{a}}$ & High SES & $p^{\mathrm{b}, \mathrm{c}, \mathrm{d}}$ \\
\hline \multicolumn{8}{|c|}{ BMI, mean (SD) } \\
\hline Age 13 & 82 & $18.6(2.0)$ & 296 & $18.3(2.4)$ & 300 & $18.0(1.9)$ & 0.059 \\
\hline Age 14 & 91 & $19.4(2.1)$ & 288 & $19.3(2.5)$ & 297 & $18.9(2.0)$ & $0.033^{\mathrm{b}}$ \\
\hline Age 15 & 109 & $20.4(2.3)$ & 330 & $20.2(2.4)$ & 315 & $20.0(2.1)$ & 0.222 \\
\hline Age 18 & 65 & $22.1(2.7)$ & 237 & $21.9(2.8)$ & 277 & $21.4(2.2)$ & $0.024^{\mathrm{b}}$ \\
\hline Age 19 & 57 & $22.5(2.6)$ & 212 & $22.2(2.8)$ & 251 & $21.8(2.3)$ & 0.154 \\
\hline Age 21 & 58 & $23.3(3.0)$ & 185 & $22.6(3.1)$ & 227 & $22.3(2.4)$ & $0.050^{\mathrm{c}}$ \\
\hline Age 23 & 56 & $23.8(3.3)$ & 194 & $23.1(3.4)$ & 231 & $22.9(2.9)$ & 0.185 \\
\hline Age 30 & 42 & $25.4(3.8)$ & 180 & $25.1(4.0)$ & 185 & $23.9(2.9)$ & $0.003^{\mathrm{c}}$ \\
\hline \multicolumn{8}{|c|}{$\begin{array}{l}\text { Overweight/ } \\
\text { obese, \% (n) }\end{array}$} \\
\hline Age 13 & 82 & $4.9(4)$ & 296 & $6.8(20)$ & 300 & $2.0(6)$ & 0.018 \\
\hline Age 14 & 91 & $6.6(6)$ & 288 & $7.3(21)$ & 297 & $2.7(8)$ & 0.035 \\
\hline Age 15 & 109 & $10.1(11)$ & 330 & $9.7(32)$ & 315 & $4.1(13)$ & 0.014 \\
\hline Age 18 & 65 & $10.8(7)$ & 237 & $11.4(27)$ & 277 & $5.4(15)$ & 0.041 \\
\hline Age 19 & 57 & $17.5(10)$ & 212 & $12.3(26)$ & 251 & $8.8(22)$ & 0.131 \\
\hline Age 21 & 58 & $22.4(13)$ & 185 & $20.0(37)$ & 227 & 11.9 (27) & 0.036 \\
\hline Age 23 & 56 & $26.8(15)$ & 194 & $25.8(50)$ & 231 & $21.2(49)$ & 0.461 \\
\hline Age 30 & 42 & $54.8(23)$ & 180 & $47.2(85)$ & 185 & $32.4(61)$ & 0.003 \\
\hline
\end{tabular}

increase in BMI of $[-0.15(95 \%$ CI $-0.32,0.01)$, $p=0.072]$. There were no substantially different results between model $3 \mathrm{a}, \mathrm{b}$ and $\mathrm{c}$, therefore, only the fully adjusted model (numbered model 3), including the complete set of the behavioural covariates, is presented in Table 3. The strongest association between SES and change in BMI at the 90th percentile observed in model 2 remained, indicating a smaller increase in BMI per year $[-0.16(95 \% \mathrm{CI}-0.32,0.01) p=0.07]$ having a high vs low SES. Figure 1 illustrates the relationship between SES and change in BMI at the 10th and 90th percentile over time. Gender did not moderate any associations between SES and change in BMI in any of the percentiles.

\section{Discussion}

The main results from this study indicate a pattern of continuous increase in average BMI over time in both genders. However, at age 30, the proportion of overweight including obese was substantially higher among males than females. Social gradients at age 30 for both average BMI and the overweight/obesity prevalence were observed. The quantile regression analyses demonstrate that both the initial and average increase in BMI over time were higher in the upper vs lower percentiles, but there were no significant association between SES and change in BMI in any parts of the BMI distribution.

The Young Hunt study from Nord Trøndelag County in mid-Norway compared two cohorts 30 years apart. They found a prevalence of overweight including obesity among adolescents (age 14-18) of $18 \%$ in the last cohort (1995-1997) compared to $10 \% 30$ years earlier (Bjornelv et al. 2009). Height and weight were measured objectively and somewhat later than the adolescence data in the NLHB study, which may partly explain the lower prevalence rates seen in our study. However, in the Hunt 3 study, conducted in 2006-2008 among adults $(n \approx 5000), 49 \%$ of the men age 20-29 were overweight or obese (Midthjell et al. 2013). This is quite comparable to the estimate of $51 \%$ among the 30-year-old men in NLHB study. The corresponding number for women in the Hunt3 study was $39 \%$ (Midthjell et al. 2013), a somewhat higher estimate than the $32 \%$ seen in our study. Parental data from 2007 from a large intervention study in the Southeastern part of Norway shows, in line with our study, an overweight/obesity prevalence based on self-report of $30 \%$ among women, and a somewhat higher prevalence of $59 \%$ among men (mean age 41 and 43, respectively). Taken together, our 


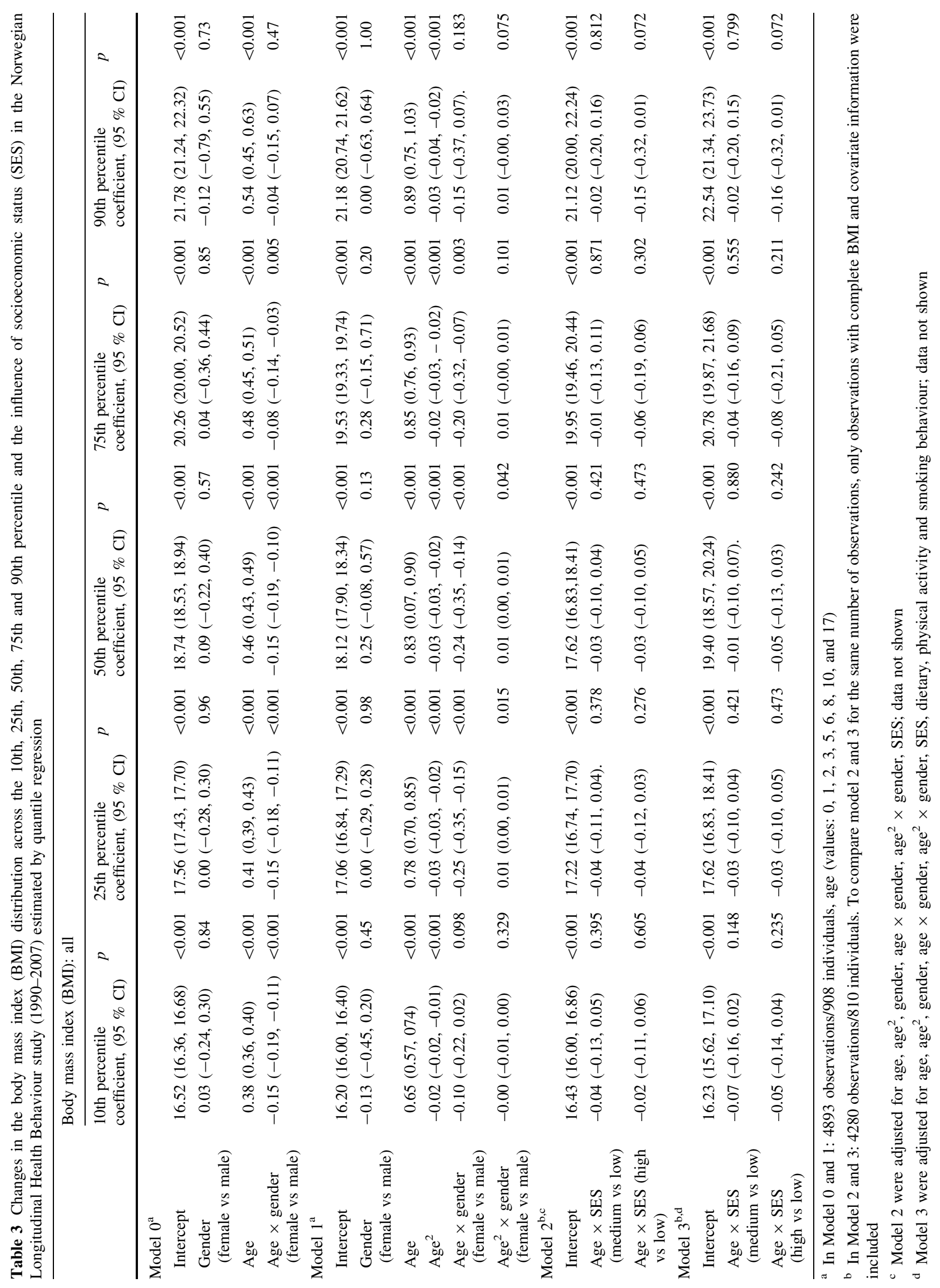




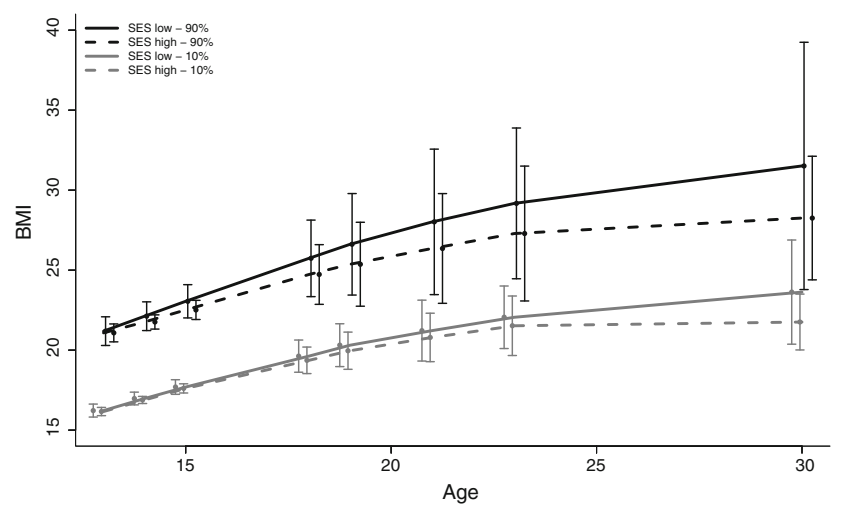

Fig. 1 10th and 90th percentiles of body mass index (BMI) for low and high socioeconomic status (SES) in the Norwegian Longitudinal Health Behaviour study (1990-2007). The BMI percentile values are based on estimates from quantile regression (model 3)

study and previous Norwegian data point to an increase in BMI taking place after the adolescence years, with evidence for a substantial increase happening throughout young adulthood (Cuypers et al. 2012).

Furthermore, when modelling change in BMI in our study, the change in BMI was greatest among those in the highest percentile, which suggests that the largest weight gain is occurring among those with the highest BMI. Even though we saw a weight gain over time in all percentiles, the result supports that the increase in overweight and obesity in recent decades may be a consequence of changes in the upper end of the BMI distribution (Ekblom et al. 2004; Kautiainen et al. 2002).

Our descriptive data indicated a development of a social gradient between the low and high adolescent SES groups over time, while the quantile regression analyses showed no significant association between adolescent SES and change in weight development across the BMI distribution. Hardy et al. (2000) examined the association between childhood SES and change in BMI during adult years. They found a relationship between father's occupation and weight gain, which increased from age 20 to 43 . Thus, there might have been evidence for the influence of adolescent SES on change in BMI in our study if the cohort had been followed up longer into adulthood. Another European study also found an association between father's occupation and change in BMI during adulthood, but for women only (Giskes et al. 2008). In our study, however, the association between adolescent SES and change in BMI did not vary by gender in any part of the BMI distribution.

Results from other longitudinal studies among adults examining SES and change in BMI show more consistent results when using "occupation" as an indicator vs "education" (Ball and Crawford 2005). However, parental education level was used as the SES indicator in this study as it seems to be a better indicator than parental income in western developed countries (Shrewsbury and Wardle 2008). It shows the strongest association with weight status among Scandinavian adolescents (Juliusson et al. 2010; Lien et al. 2007; Matthiessen et al. 2014), and is assumed to stay relatively stable over time. A large 11-year study among adults (age 20-79) from two regions in Norway, conducted about the same time period as our study (1990-2001), showed in consistence with the findings in the NLHB study that weight gain occurred across all education and income brackets with no differential associations between SES and change in BMI (Reas et al. 2007).

There may be several explanations for our finding of no association between adolescent SES and weight development. The rapid development of an obesogenic environment over the last decades could possibly have contributed to a narrowing of the socioeconomic gap in the BMI distribution (Zhang and Wang 2004). Another hypothesis suggests that education level in general may be more strongly related to weight and obesity prevalence when other socioeconomic resources are low (Mirowsky and Ross 2003). In contradiction, Norway is a relatively high-income country with relative small average difference between those "less and better well off".

The long follow-up period with participants from late childhood to middle adulthood is the main strength of this study. In addition, the parental education measure was based on reports taken during the adolescent years and should not be affected by recall bias to the same extent as in other studies using retrospective recall (Galobardes et al. 2006; Giskes et al. 2008). However, the predictive value of the SES variable may have been reduced because it was constructed from both parents' and adolescents' reports of parental educational level, and the congruence between adolescents' and parents' report has previously been found to only be fair (Lien et al. 2001a). In addition, some parents may have improved their education level between the start of the study in 1990 and 1996, when they reported their education level. Males and those with low adolescent SES were underrepresented due to nonresponse after age 18. Self-reported height tends to be over-reported and weight under-reported, especially among the heaviest and those adolescents who regard themselves as more fat (Connor Gorber et al. 2007; Flood et al. 2000; Jansen et al. 2006). Therefore, the BMI levels and overweight/obesity prevalences are probably underestimated in our study. Among adolescents underestimation is found to be higher in low SES groups as well (Jansen et al. 2006). Hence, biases related to both drop-out and self-reported BMI may have caused an attenuation of the association between SES and change in BMI. Furthermore, we have not been able to adjust for the participants' own education level as adults because the 
decline in participation by age 30 caused a high number of missing for this variable. Including two probably highly collinear variables to a regression model could, however, lead to invalid conclusions (Galobardes et al. 2006). We recommend that further studies should examine both childhood and adulthood SES, and also assess whether change in SES throughout the life course influences weight development.

In conclusion, despite the limitations, this study indicates that those in the upper BMI percentile are those at most risk of gaining weight and might be more prone to the influence of SES on weight gain over time. Targeted strategies to reach those with the highest risk of developing an unhealthy weight seem needed, while inequalities in weight gain should be further explored with better measures of both BMI and SES.

Acknowledgments The NLHB study was initiated and conducted from the Department of Health Promotion and Development, University of Bergen, and is currently coordinated by professor Bente Wold. KIK founded the study. NL and IHB conceived the idea for the paper. AA and IHB prepared the data. $\varnothing \mathrm{S}$ and IHB carried out the analyses. All authors provided feedback and had final approval of the submitted and published versions. The Norwegian Research Council is the main funding source of the study. The authors have no competing interests.

Open Access This article is distributed under the terms of the Creative Commons Attribution 4.0 International License (http:// creativecommons.org/licenses/by/4.0/), which permits unrestricted use, distribution, and reproduction in any medium, provided you give appropriate credit to the original author(s) and the source, provide a link to the Creative Commons license, and indicate if changes were made.

\section{References}

Anderssen N, Jacobs DR Jr, Aas H, Jakobsen R (1995) Do adolescents and parents report each other's physical activity accurately? Scand J Med Sci Sports 5(5):302-307

Ball K, Crawford D (2005) Socioeconomic status and weight change in adults: a review. Soc Sci Med 60(9):1987-2010. doi:10.1016/ j.socscimed.2004.08.056

Berghofer A, Pischon T, Reinhold T, Apovian CM, Sharma AM, Willich SN (2008) Obesity prevalence from a European perspective: a systematic review. BMC Public Health 8:200. doi:10.1186/1471-2458-8-200

Bjelland $\mathrm{M}$ et al (2010) Overweight and waist circumference among Norwegian 11-year-olds and associations with reported parental overweight and waist circumference: the HEIA study. Scand J Public Health 38(5 Suppl):19-27. doi:10.1177/140349481038 5036

Bjornelv S, Lydersen S, Holmen J, Lund Nilsen TI, Holmen TL (2009) Sex differences in time trends for overweight and obesity in adolescents: the Young-HUNT study. Scand J Public Health 37(8):881-889. doi: $10.1177 / 1403494809347022$

Bottai $\mathrm{M}$ et al (2014) Use of quantile regression to investigate the longitudinal association between physical activity and body mass index. Obesity (Silver Spring, Md) 22(5):E149-E156. doi:10. 1002/oby.20618
Brisbois TD, Farmer AP, McCargar LJ (2012) Early markers of adult obesity: a review. Obes Rev 13(4):347-367. doi:10.1111/j.1467789X.2011.00965.x

Brug J et al (2012) Differences in weight status and energy-balance related behaviors among schoolchildren across Europe: the ENERGY-project. PLoS One 7(4):e34742. doi:10.1371/journal. pone. 0034742

Cole TJ, Lobstein T (2012) Extended international (IOTF) body mass index cut-offs for thinness, overweight and obesity. Pediatr Obes 7(4):284-294. doi:10.1111/j.2047-6310.2012.00064.x

Connor Gorber S, Tremblay M, Moher D, Gorber B (2007) A comparison of direct vs. self-report measures for assessing height, weight and body mass index: a systematic review. Obes Rev 8(4):307-326. doi:10.1111/j.1467-789X.2007.00347.x

Cuypers K, Kvaloy K, Bratberg G, Midthjell K, Holmen J, Holmen TL (2012) Being normal weight but feeling overweight in adolescence may affect weight development into young adulthood-an 11-year followup: the HUNT Study, Norway. J Obes 2012:601872. doi: $10.1155 / 2012 / 601872$

Dietz WH (1994) Critical periods in childhood for the development of obesity. Am J Clin Nutr 59(5):955-959

Ekblom O, Oddsson K, Ekblom B (2004) Prevalence and regional differences in overweight in 2001 and trends in BMI distribution in Swedish children from 1987 to 2001. Scand J Public Health 32(4):257-263. doi:10.1080/1403494031009498

Flood V, Webb K, Lazarus R, Pang G (2000) Use of self-report to monitor overweight and obesity in populations: some issues for consideration. Aust N Z J Public Health 24(1):96-99

Friestad C, Klepp K-L (1997) Social influences on the development of boys' and girls' smoking behavior. J Gend Cult Health 2:287-304

Galobardes B, Smith GD, Lynch JW (2006) Systematic review of the influence of childhood socioeconomic circumstances on risk for cardiovascular disease in adulthood. Ann Epidemiol 16(2):91-104. doi:10.1016/j.annepidem.2005.06.053

Giskes K, van Lenthe FJ, Turrell G, Kamphuis CB, Brug J, Mackenbach JP (2008) Socioeconomic position at different stages of the life course and its influence on body weight and weight gain in adulthood: a longitudinal study with 13-year follow-up. Obesity (Silver Spring, Md) 16(6):1377-1381. doi:10. 1038/oby.2008.54

Hao L, Naiman DQ (2007) Quantile regression, vol 07-149. Sage, Thousand Oaks, Calif., p 126

Hardy R, Wadsworth M, Kuh D (2000) The influence of childhood weight and socioeconomic status on change in adult body mass index in a British national birth cohort. Int J Obes Relat Metab Disord 24(6):725-734

Jansen W, van de Looij-Jansen PM, Ferreira I, de Wilde EJ, Brug J (2006) Differences in measured and self-reported height and weight in Dutch adolescents. Ann Nutr Metab 50(4):339-346. doi:10.1159/000094297

Juliusson PB, Eide GE, Roelants M, Waaler PE, Hauspie R, Bjerknes $R$ (2010) Overweight and obesity in Norwegian children: prevalence and socio-demographic risk factors. Acta Paediatr 99(6):900-905. doi:10.1111/j.1651-2227.2010.01730.x

Kautiainen S, Rimpela A, Vikat A, Virtanen SM (2002) Secular trends in overweight and obesity among Finnish adolescents in 1977-1999. Int J Obes Relat Metab Disord 26(4):544-552

Lien N, Friestad C, Klepp KI (2001a) Adolescents' proxy reports of parents' socioeconomic status: how valid are they? J Epidemiol Community Health 55(10):731-737

Lien N, Lytle LA, Klepp KI (2001b) Stability in consumption of fruit, vegetables, and sugary foods in a cohort from age 14 to age 21 . Prev Med 33(3):217-226. doi:10.1006/pmed.2001.0874

Lien N, Kumar BN, Holmboe-Ottesen G, Klepp KI, Wandel M (2007) Assessing social differences in overweight among 15- to 
16-year-old ethnic Norwegians from Oslo by register data and adolescent self-reported measures of socio-economic status. Int $\mathbf{J}$ Obes (Lond) 31(1):30-38. doi:10.1038/sj.ijo.0803415

Matthiessen J, Stockmarr A, Biltoft-Jensen A, Fagt S, Zhang H, Groth MV (2014) Trends in overweight and obesity in Danish children and adolescents: 2000-2008-exploring changes according to parental education. Scand J Public Health 42(4):385-392. doi: $10.1177 / 1403494813520356$

Meyer HE, Tverdal A (2005) Development of body weight in the Norwegian population. Prostaglandins Leukot Essent Fatty Acids 73(1):3-7. doi:10.1016/j.plefa.2005.04.003

Midthjell $\mathrm{K}$ et al (2013) Trends in overweight and obesity over 22 years in a large adult population: the HUNT Study, Norway. Clin Obes 3(1-2):12-20. doi:10.1111/cob.12009

Mirowsky J, Ross CE (2003) Education, social status, and health. Walter de Gruyter, New York

Mitchell JA, Pate RR, Beets MW, Nader PR (2013a) Time spent in sedentary behavior and changes in childhood BMI: a longitudinal study from ages 9 to 15 years. Int $\mathbf{J}$ Obes (Lond) 37(1):54-60. doi:10.1038/ijo.2012.41

Mitchell JA, Pate RR, Espana-Romero V, O’Neill JR, Dowda M, Nader PR (2013b) Moderate-to-vigorous physical activity is associated with decreases in body mass index from ages 9 to 15 years. Obesity (Silver Spring, Md) 21(3):E280-E293. doi:10. 1002/oby.20118

Mitchell JA, Rodriguez D, Schmitz KH, Audrain-McGovern J (2013c) Greater screen time is associated with adolescent obesity: a longitudinal study of the BMI distribution from ages 14 to 18 . Obesity (Silver Spring, Md) 21(3):572-575. doi:10. 1002/oby.20157
Pate RR et al (2013) Factors associated with development of excessive fatness in children and adolescents: a review of prospective studies. Obes Rev 14(8):645-658. doi:10.1111/obr. 12035

Power C, Lake JK, Cole TJ (1997) Measurement and long-term health risks of child and adolescent fatness. Int J Obes Relat Metab Disord 21(7):507-526

Reas DL, Nygard JF, Svensson E, Sorensen T, Sandanger I (2007) Changes in body mass index by age, gender, and socio-economic status among a cohort of Norwegian men and women (19902001). BMC Public Health 7:269. doi:10.1186/1471-2458-7-269

Shrewsbury V, Wardle J (2008) Socioeconomic status and adiposity in childhood: a systematic review of cross-sectional studies 1990-2005. Obesity (Silver Spring, Md) 16(2):275-284. doi:10. 1038/oby.2007.35

Tamayo T, Christian H, Rathmann W (2010) Impact of early psychosocial factors (childhood socioeconomic factors and adversities) on future risk of type 2 diabetes, metabolic disturbances and obesity: a systematic review. BMC Public Health 10:525. doi:10.1186/1471-2458-10-525

Wei Y, Pere A, Koenker R, He X (2006) Quantile regression methods for reference growth charts. Stat Med 25(8):1369-1382. doi:10. $1002 /$ sim. 2271

World Health Organization (WHO) (2000) Obesity: preventing and managing the global epidemic. Report of a WHO Consultation. WHO technical report series 894. WHO, Geneva

Zhang Q, Wang Y (2004) Trends in the association between obesity and socioeconomic status in U.S. adults: 1971 to 2000 . Obes Res 12(10):1622-1632. doi:10.1038/oby.2004.202 